\title{
Superior Dislocation of the Patella in a Young Woman without Osteophytes: A Case Report
}

\author{
Tatsunori Kataoka, Norishige lizawa and Shinro Takai \\ Department of Orthopaedic Surgery, Nippon Medical School
}

\begin{abstract}
Superior dislocation of the patella without patellar ligament injury is an extremely rare condition. A review of the English-language literature found only 23 reported cases. In addition, the primary factor for dislocation in most of these cases was considered to be osteophytes in the inferior pole of the patella and the anterior surface of the femoral condyle; only 1 case had no osteophytes. We treated a 19-yearold woman who sustained a painful locking of the left knee after colliding with a friend. Plain radiography and computed tomography showed superior-lateral dislocation of the patella and an interlocking between notches in the inferior pole of the patella and the anterior surface of the femoral condyle. Closed reduction without sedation was performed without difficulty, and the patient was able to walk home without pain. After 1 week, the knee was without problems. The patient had no osteophytes in the knee and had no other common risk factors, such as patella alta, ligamentous laxity, genu recurvatum, and paralytic disorders. After a comparison with previously reported cases of superior patella dislocation, we concluded that the primary factor in the present case might have been a different condition. (J Nippon Med Sch 2016; 83: 24-26)
\end{abstract}

Key words: patella, superior dislocation, notch

\section{Introduction}

Among traumatic patellar dislocations, superior dislocation without patellar ligament injury is extremely rare. A review of the English-language literature found only 23 reported cases ${ }^{1-12}$. In addition, we found only 1 reported case of superior dislocation of the patella without osteophytes ${ }^{7}$.

We treated a case of superior dislocation of the patella without osteophytes or other known risk factors. Accordingly, we believe that a primary factor for superior patella dislocation might be a different condition.

\section{Case Report}

A 19-year-old women sustained painful locking of the left knee after colliding with a friend while dancing at school. She fell to the ground, and the top of the left knee was fallen upon by the friend. While being examined she had anterior knee pain and was unable to bend the knee. Plain radiography of the knee showed superior dislocation of the patella without fracture or arthrosis
(Fig. 1). Computed tomography showed superior-lateral dislocation of the patella and notches in the inferior pole of the patella and the anterior surface of the femoral condyle (Fig. 2 and 3), which prevented the return of the patella. Closed reduction without sedation was performed without difficulty. The knee pain resolved after reduction, and plain radiography showed normal alignment of the knee (Fig. 4). The Insall-Salvati ratio was 1, and no patella alta was observed. The patient was able to bend the knee normally and walk home. One week later, she had no problem with the knee and no effusion or tenderness of the patellar ligament.

\section{Discussion}

Superior dislocation of the patella without patellar ligament injury was first reported by Watson-Jones in 1956, and to date only 23 cases have been reported in the English-language literature. However, only 1 of these reported cases was without osteophytes. To our knowledge, our present patient is the youngest to have supe-

Correspondence to Tatsunori Kataoka, MD, Department of Orthopaedic Surgery, Nippon Medical School, 1-1-5 Sendagi, Bunkyo-ku, Tokyo 113-8602, Japan

E-mail: 1109tk1986@nms.ac.jp

Journal Website (http://www.nms.ac.jp/jnms/) 


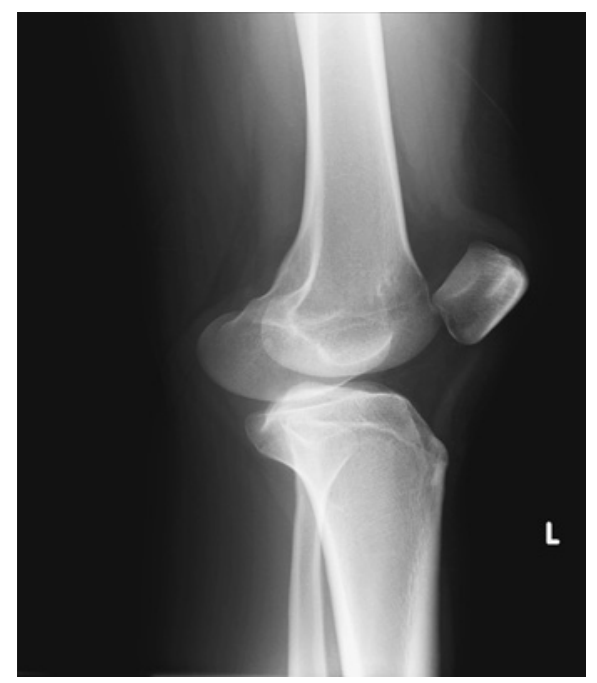

Fig. 1 Lateral radiography showing superior dislocation of the patella without any osteophytes.

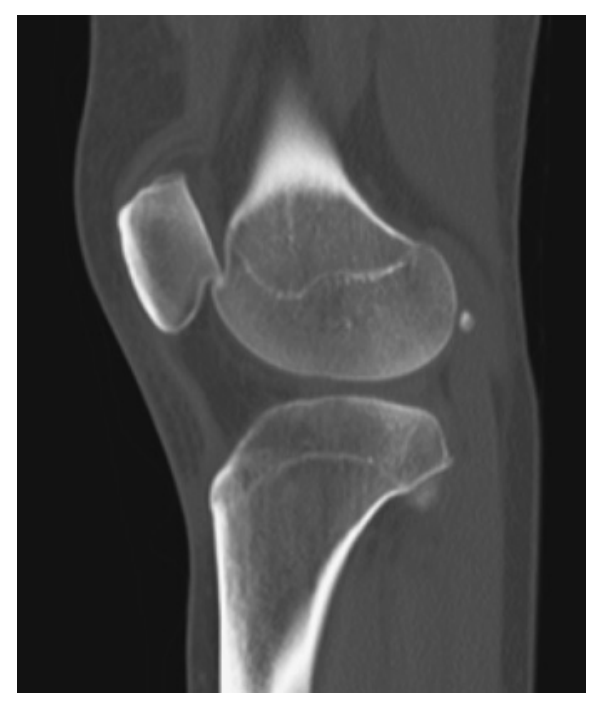

Fig. 2 Computed tomography showing superior dislocation of the patella without any osteophytes.

rior dislocation of the patella and the only patient to exhibit no recognized risk factors.

Direct impact to the lower aspect of the patella and hyperextension of the knee are the usual mechanisms for superior dislocation of the patella, and pain and locked in extension of the knee are the common symptoms. Hanspal has reported a similar case caused by direct impact $^{4}$, and Teuscher and Goletz have reported a similar case caused by atraumatic hyperextension ${ }^{10}$. In the present case, both mechanisms were involved.

The most common treatment for superior dislocation of the patella is closed reduction with or without sedation, which can usually be performed without difficulty. After reduction the patient is usually free of pain and compli-

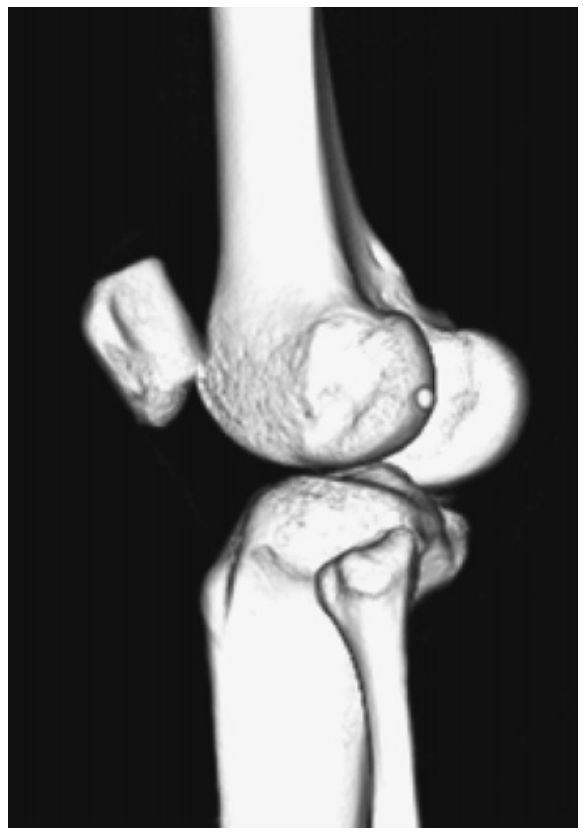

Fig. 3 Three-dimensional computed tomography showing superior dislocation of the patella without any osteophytes.

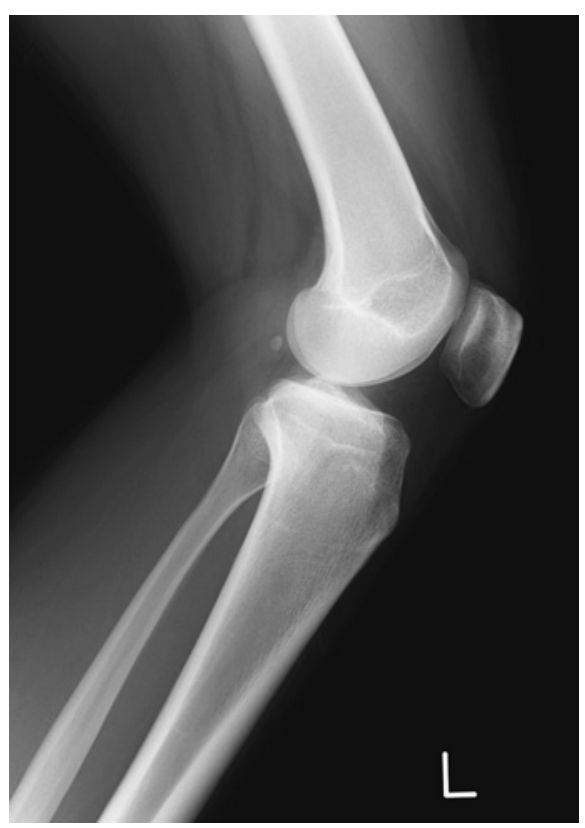

Fig. 4 Lateral radiography showing normal alignment of the knee with an Insall-Salvati ratio of 1.

cations. However, Takai et al. and Hansen et al. have reported cases with complications, such as recurrence, which resolved after arthroscopic treatment ${ }^{3,9}$. In our case, closed reduction without sedation was performed. We hyperextended the knee and moved the patella upward to release the interlock between the patella and the anterior surface of the femoral condyle and then shifted the 
patella to the appropriate position. Because there were no osteophytes or complications, arthroscopic treatment was not performed.

The risk factors of superior dislocation of the patella reported by Teusher and Goletz ${ }^{10}$ are patella alta, ligamentous laxity, genu recurvatum, and paralytic disorders. Among the 23 cases reported in the Englishlanguage literature, all but 1 had patella alta (InsallSalvati ratio $>1.20$ ). Because none of these risk factors were present in our patient, we believe that the primary factor might have been a different condition. One factor that we considered was notches found on the inferior pole of the patella and the anterior surface of the femoral condyle. When notches are discovered in such locations, they should be considered a risk factor for superior patella dislocation.

The cause of these notches was not clear, but we considered that they were congenital or were caused by microfractures or cartilage injuries on the inferior pole of the patella and the anterior surface of the femoral condyle. However, computed tomography revealed no ossicles, and the patient had no pain 1 week later. Therefore, we performed no further examinations.

Superior dislocation of the patella is a rare injury most often found in elderly patients and more rarely found in younger patients. Various risk factors, usually agerelated, have been reported, but in some cases, risk factors are not apparent. Therefore, it is important to be aware of this clinical problem in elderly persons with osteophytes and in young persons with no apparent risk factors.

Conflict of Interest: The authors declare no conflict of interst.

\section{References}

1. Bassi RS, Kumar BA: Superior dislocation of the patella; a case report and review of the literature. Emerg Med J 2003; 20: 97-98.

2. Gakhar H, Singhal A: Superior dislocation of the Patella: Case report and Review of the Literature. J Emerg Med 2013; 44: 478-480.

3. Hansen B, Beck C, Townsley R: Arthroscopic Removal of a Loose Body Osteophyte Fragment After Superior Patellar Dislocation With Locked Osteophytes. Arthroscopy 2003; 19: E25.

4. Hanspal RS: Superior dislocation of the patella. Injury 1985; 16: 487-488.

5. Mashfiqul A, Siddiqui M, Tan H: Locked knee from superior dislocation of the patella-diagnosis and management of a rare injury. Knee Surg Sports Traumatol Arthrosc 2011; 19: 671-673.

6. Ofluoglu O, Yasmin D, Donthineni R, Yildiz M: Superior dislocation of the patella with early onset patellofemoral arthritis: a case report and literature review. Knee Surg Sports Traumatol Arthrosc 2006; 14: 350-355.

7. Saleemi AJ, Hussain A, Iqbal MJ, Thuse MG, George AA: Superior dislocation of patella in a rugby player: an update on a extremely rare condition and review of literature. Knee Surg Sports Traumatol Arthrosc 2007; 15: 11121113.

8. Scott SJ, Molloy A, Harvey RA: Superior dislocation of the patella- a rare but important differential diagnosis of acute knee pain - a case report and review of the literature. Injury 2000; 31: 543-545.

9. Takai S, Yoshino N, Hirasawa Y: Arthroscopic treatment of voluntary superior dislocation of the patella. Arthroscopy 1998; 14: 753-756.

10. Teuscher DD, Goletz TH: Recurrent atraumatic superior dislocation of the patella: case report and review of the literature. Arthroscopy 1992; 8: 541-543.

11. Wood L, Stirrat AN: Superior dislocation of the patella: a case report and review of the literature. Knee 1998; 5: 299-300.

12. Yip DKH, Wong JWK, Sun LK, Wong NM, Chan CW, Lau PY: The management of superior dislocation of the patella with interlocking osteophytes - an update on a rare problem. J Orthop Surg (Hong Kong) 2004; 12: 254-257.

(Received, February 11, 2015)

(Accepted, June 9, 2015) 